\title{
Euler-Bernoulli Beam Theory in the Presence of Fiber Bending Stiffness
}

\author{
${ }^{1}$ Ali Farhat, ${ }^{2}$ NbilaGwila \\ ${ }^{I}$ Al-Asmarya Islamic UniversityFaculty of Science-ZlitenMathematics department \\ ${ }^{2}$ Al-Asmarya Islamic UniversityMathematics departmentFaculty of Science-Zliten
}

\begin{abstract}
: in the conventional one-dimensional mathematical modelling of fibre-reinforced thin-walled structures it is assumed that fibres have negligible thickness and they are perfectly flexible. Consequently, the constitutive equation forfinitedeformations of fibre-reinforced elastic solids involves no natural length. Based on incorporatingfibre bending stiffness into a continuum theory, asymmetric linearised elasticity theory that takes into consideration the effects of fibres bending stiffness, has been produced in[1]. Relatively, an accurate $2 d$ thin-walled structures modeling has been presented in [2]. Based on the aforementioned $2 d$-theory and, as a particular case, this study presents one-dimensional mathematical modeling that takes into consideration the fibres bending stiffness, with the use of the displacement approximation of the bernoulli-euler beam model. Unlike its conventional one-dimensionalmodelingthe solution is obtained that contains an intrinsic material length parameter which may, for instance, be considered representative of thefibre diameter. Numerical results on the basis of the obtained solution are presented and discussed in this article for different boundary conditions of homogeneous fibre-reinforced rectangular beams.
\end{abstract}

Keywords:fiber bending stiffness, beam deflection, couple-stress, asymmetric elasticity, intrinsic material area or length parameter.

\section{Introduction}

The presence of the fiber bending stiffness has received a significant attention that appears in the literature for studying the continuum theory of finite deformations of elastic materials. A non-symmetric stress and the couple-stress are requiredinasymmetric elasticity theory presented in [1]. Relatively, a version of asymmetric linear elasticity theory has been produced, as a particular case, by Spencer and Soldatos[1].A generalized, 6-degree-of-freedom, 2D model for plates with fibres resistant to bending has been developed by Soldatos in [2] where one extra elastic modulus, which has dimensions of force, is involved in each layer of the laminate. Consequently, some intrinsic material area or length parameter involved in the analysis that may be associated, for instance, with fiber thickness of fiber spacing[3]. With the use of the plate displacement approximation of Reissner $(1944,1945)$, plate model that accounts for fibrethickness and, consequently, for the ability of individual fibres to resist bending[4].Based on the aforementioned plates model developed in [2], different displacement approximations, which available in the literature, can be used to produce thin elastic plate and beam models that accounts for the effects of fibre resistant in bending. One of the commune usesof the aforementioned displacement approximations is the Bernoulli-Euler beam theory that provides a means of calculating the deflection of elastic beams which deformed in linear scale. As pointed out in [5], because of the simplicity of Euler-Bernoulli beam theory that provides reasonable engineering approximations when applied on several problems, it is commonly used. In addition, this theory appears in the literature with different names such as classical beam theory, Bernoulli beam theory, or Euler beam theory. This conventional theory was applied to solve problems of small elastic beams deflection where the beams are reinforced by fibres which assumed to have negligible thickness and they are perfectly flexible. In the present study, the fibres are assumed to have the ability to resist bending. Consequently, an extra elastic modulus has been involved in the theory, which, unlike its conventional counterparts.

The purpose of the this article is to present a beam theory with the use of the displacement approximation of the Bernoulli-Euler beam model in connection with the generalized, 6-degree-of-freedom, 2Dplates model thathas been developed by Soldatos in [2]. A cross-ply laminated beam of transversely isotropic, linearly elastic material that contains a single, unidirectional family of straight fibres which can resist bending is assumed to be subjected to small deformation is considered in the present study. The Constitutive equations considering fiber bending stiffness have been produced based on the use aforementioned displacement approximation of the Bernoulli-Euler beam model in section 3. Consequently, section 4 presents the Navier-type differential equations which have been solved for the static solution in section 5. Numerical results and relevant discussionbased on the obtained solutions have been conducted in section 7. The main conclusions of this article as well as some research points for future related work are finally summarized in Section 8 . 


\section{Problem Formulation}

Consider a cross-ply laminated plate of transversely isotropic, linearly elastic material having arbitrary constant thickness $h$ in the $z$ direction and, horizontal constant length $\mathrm{L}_{1}$ in the $x$ direction. The considered plate is assumed to be of infinite extent in the $y$ direction. Furthermore, the plate contains fibers which are lying in parallel to the $x$-directionand has the ability to resist bending. Different sets of end boundary conditions are applied on the ends $x=0, L_{1}$.

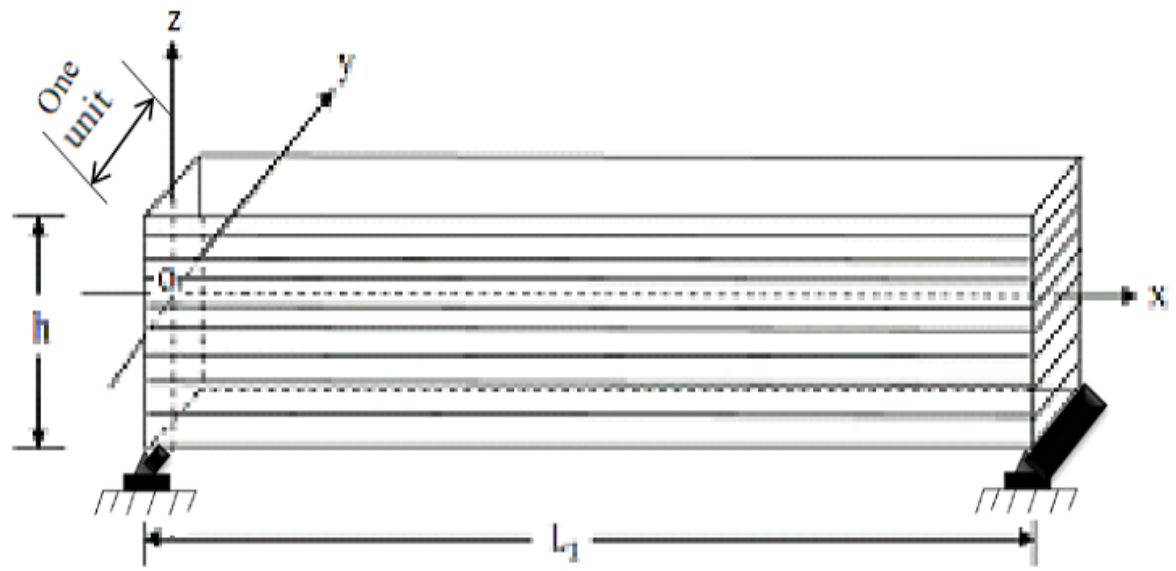

Figure1. Co-ordinate system

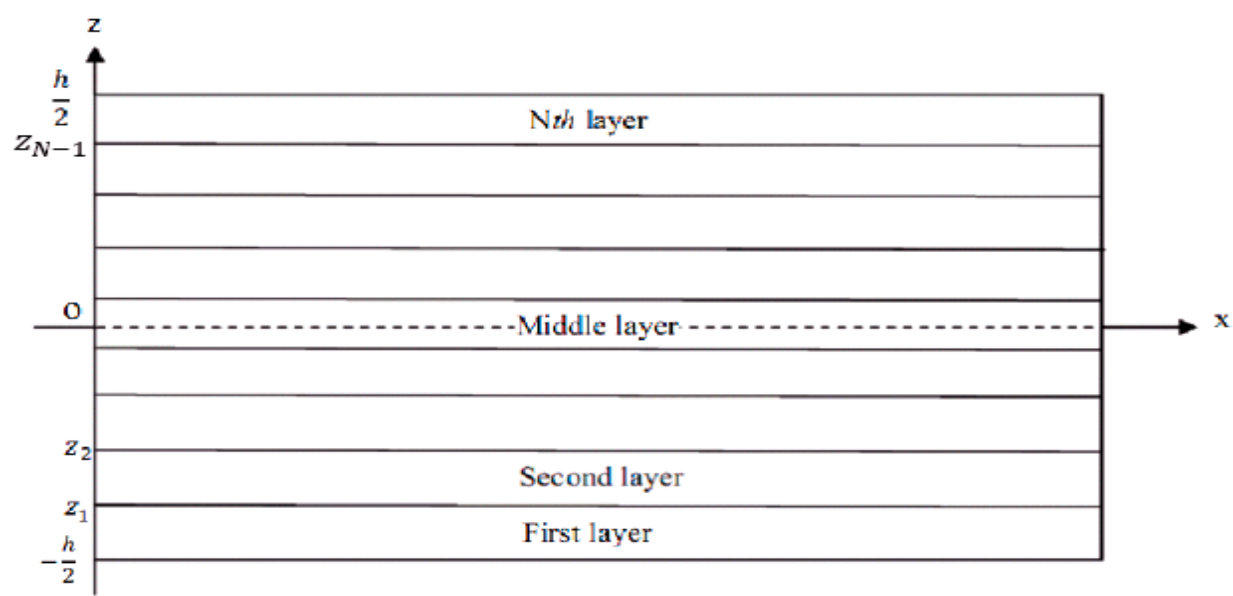

Figure 2.An example of the beam layer numbering

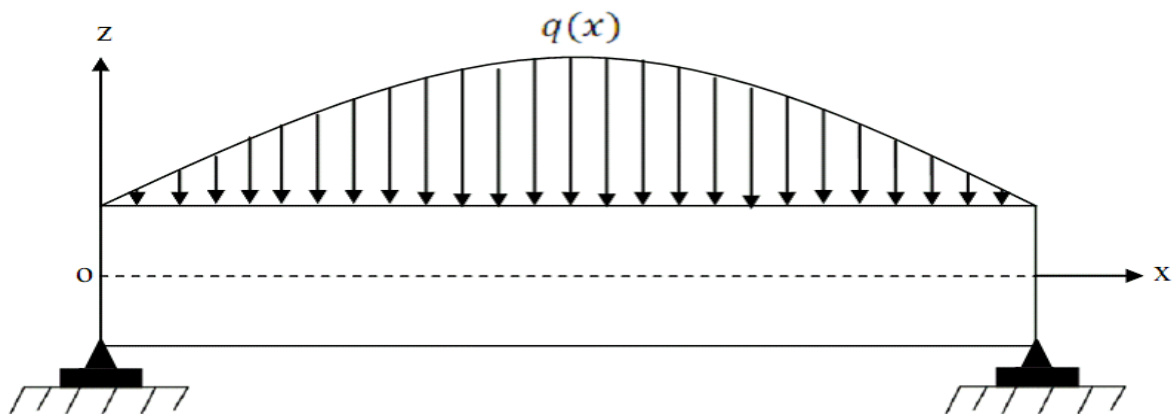

Figure 3.Lateral loading

As a result of the plane-strain symmetries involved in this problem, all quantities involved are independent of $\mathrm{y}$ and the displacement function in $y$ direction is equal to zero. According to this any of the plate cross-sections could alternatively be regarded as a transversely isotropic beam of thickness $h$, length $\mathrm{L}_{1}$ and a unit width along the $y$-direction. A given external lateral loading $q(x)$ is applied on the beam which causes small static flexure. The loading acts normally and downwards on thetop lateral plane of the beam. 


\section{Constitutive Equations Considering Fiber Bending Stiffness}

The elastic beam model begins with the displacement approximation of the Euler-Bernoulli Beam theory that has the following form:

$U(x, z, t)=u(x, t)-z w_{, x},(1-\mathrm{a})$

$W(x, z, t)=w(x, t)$

where $\mathrm{t}$ denotes time. In addition, $\mathrm{U}(\mathrm{x}, \mathrm{z}, \mathrm{t})$ and $\mathrm{W}(\mathrm{x}, \mathrm{z}, \mathrm{t})$ represent the displacement components of a point $(\mathrm{x}, \mathrm{y}, \mathrm{z}, \mathrm{t})$ along $x$ and $z$ directions, respectively. Moreover, $w(x, t)$ presents the transverse deflection of the beam. It can be seen that the transverse deflection is assumed to be independent of the beam thickness. In addition, $u(x . t)$ represents the in-plane displacement of the beam middle plane. Because of that, they have evidently dimension of length.

Inserting the displacement approximation (1) into the following well-known linear kinematic relations:

$\varepsilon_{x}=\frac{\partial U}{\partial x}, \gamma_{x z}=\frac{\partial U}{\partial z}+\frac{\partial W}{\partial x}$,

yields the following approximate strain field:

$\varepsilon_{x}=e_{x}^{c}+z k_{x}^{c}, \gamma_{x z}=0$,

where

$e_{x}^{c}=u_{, x}$, and $k_{x}^{c}=-w_{, x x}$,

where the prime denotes the ordinary differentiation with respect to $z$. The quantities denoted with a superscript "c" are identical with their classical beam theory counterparts where the fibers have no bending stiffness.

The beam is assumed to be composed of an arbitrary number, $N$, of perfectly bonded transversely isotropic layers. Accordingly, the generalised Hooke's law within the $k$ th layer of such a cross-ply laminate is given as follows:

$\sigma_{x}^{(k)}=Q_{11}^{(k)} \varepsilon_{x}, \tau_{(x z)}^{(k)}=Q_{55}^{(k)} \gamma_{x z}=Q_{55}^{(k)}(0)=0$,

where $\tau_{(x z)}$ denotes to the symmetric part of the stress tensor component and the appearing $Q^{s}$ are the reduced stiffnesses [6]. Quantities with superscript " $k$ " are due to the $k^{\text {th }}$ layer of a cross-ply laminate.

In the presence of the fiber bending stiffness, the elasticity theory requires to include the couple-stress and consequently asymmetric stress [1]. The anti-symmetric part of the associated shear stress tensor component is denoted by symbol with indices in square brackets. This part is defined as follows[2]:

$\tau_{[x z]}^{(k)}=-\tau_{[z x]}^{(k)}=\frac{1}{2} m_{x y, x}^{(k)}=\frac{1}{2} d^{f^{(k)}} K_{z, x}^{f}=-\frac{1}{2} d^{f^{(k)}} w_{, x x x}$.

Here $K_{z}^{f^{(k)}}$ represents the fibers curvature.In addition, $m_{x y}^{(k)}$ represents the non-zero couple stress which is related to the fiber curvature [2]. Moreover, $d^{f^{(k)}}$ is an elastic modulus that accounts for the fiber bending stiffness in the $k$-thlayer. This elastic modulus has dimension of force [2]. In this regard, the following notation:

$d^{f^{(k)}}=C_{11} l^{(k)} L_{1}$,

is employed to include a material intrinsic length parameterl $l^{(k)}$, which may, for instance, be considered to be related to the fiber thickness in the $k$-th layer[3]. Thus, the shear stresses take the following form:

$\tau_{x z}^{(k)}=\tau_{(x z)}^{(k)}+\tau_{[x z]}^{(k)}$,

$\tau_{z x}^{(k)}=\tau_{(x z)}^{(k)}-\tau_{[x z]}^{(k)}$,

The equations of motion [2] take the following form:

$\sigma_{x, x}^{(k)}+\tau_{(x z), z}^{(k)}-\tau_{[x z], z}^{(k)}=\rho \ddot{U}^{(k)}$,

$\tau_{(x z), x}^{(k)}+\tau_{[x z], x}^{(k)}+\sigma_{z, z}^{(k)}=\rho \ddot{W}^{(k)}$.

Furthermore, with the use of equations (5), (6) and (8), the shear stresses can be written as follows:

$\tau_{x z}^{(k)}=Q_{55}^{(k)} \gamma_{x z}-\frac{1}{2} d^{f^{(k)}} w_{, x x x}=-\frac{1}{2} d^{f^{(k)}} w_{, x x x}$,

$\tau_{z x}^{(k)}=Q_{55}^{(k)} \gamma_{x z}+\frac{1}{2} d^{f(k)} w_{, x x x}=\frac{1}{2} d^{f(k)} w_{, x x x}$, 
In addition, the force and moment resultants are as follows:

$N_{x}^{c}=\int_{-\frac{h}{2}}^{\frac{h}{2}} \sigma_{x} d z, M_{x}^{c}=\int_{-\frac{h}{2}}^{\frac{h}{2}} \sigma_{x} z d z, M_{x}^{f}=\frac{1}{2} \int_{-\frac{h}{2}}^{\frac{h}{2}} m_{x y} d z$,

Where $\mathrm{M}_{\mathrm{x}}^{\mathrm{f}}$ is related to the moment resultants caused by the present of the fiber bending stiffness. In order to obtain the tow unknown degrees of freedom $(u$, and $w)$, the following relevant equations of motion (Soldatos, 2009) will be applied:

$N_{x, x}^{c}=\rho_{0} \ddot{u}-\rho_{1} \ddot{w}_{, x}$,

$M_{x, x x}^{c}+M_{x, x x}^{f}=q(x)+\rho_{0} \ddot{w}+\rho_{1} \ddot{u}_{, x}-\rho_{2} \ddot{w}_{, x x}$,

where the dots stand for ordinary differentiation with respect to time and, the coefficients which are in the inertia terms are defined as follows:

$\rho_{i}=\int_{-\frac{h}{2}}^{\frac{h}{2}} \rho z^{i} d z$.

Here $\rho$ denotes the material density of the elastic beam considered. The equations of motion (13) are associated with the following variationally consistent set of end boundary conditions at $x=0, L_{1}$ [2]:

either $u$ or $N_{x}^{c}$ is prescribed,

eitherw or $M_{x, x}^{c}+M_{x, x}^{f}$ is prescribed,

eitherw $w_{x}$ or $M_{x}^{c}+M_{x}^{f}$ is prescribed,

\section{Navier-Type Differential Equations}

Inserting the equations (5-8) and (10) into equations (11) yields the following force and moment resultants in terms of the tow degrees of freedom and their derivatives:

$\left[\begin{array}{l}N_{x}^{c} \\ M_{x}^{c}\end{array}\right]=\left[\begin{array}{ll}A_{11}^{c} & B_{11}^{c} \\ B_{11}^{c} & D_{11}\end{array}\right] \times\left[\begin{array}{c}u_{, x} \\ -w_{, x x}\end{array}\right], M_{x}^{f}=-\frac{1}{2} h d^{f} w_{, x x}$,

where the appearing rigidities can be calculated by the use of the following expressions:

$\left(A_{11}^{c}, B_{11}^{c}\right)=\int_{-\frac{h}{2}}^{\frac{h}{2}} Q_{55}^{(k)}(1, z) d z$,

$D_{11}=\int_{-\frac{h}{2}}^{\frac{h}{2}} Q_{11}^{(k)} z^{2} d z$,

The equations of motion (12) which contain the term that related to the presence of the fiberbending stiffness can be converted into a set of tow differential equations for the same number of main unknowns as follows: With the use of equation (3.13), these differential equations can be obtained in terms of the displacement field yielding the following Navier-type differential equations system:

$A_{11}^{c} u_{, x x}-B_{11}^{c} w_{, x x x}=\rho_{0} \ddot{u}-\rho_{1} \ddot{w}_{, x}$,
$B_{11}^{c} u_{, x x x}-\left(D_{11}^{f}\right) w_{, x x x x}=q(x)+\rho_{0} \ddot{w}+\rho_{1} \ddot{u}_{, x}-\rho_{2} \ddot{w}_{, x x}$,

which are called the Navier-type differential equations system where the shown rigidity in equation (3.16-b) is depending on the conventional rigidity, $\mathrm{D}_{11}$, and the fiber bending elastic modulus $\mathrm{d}^{\mathrm{f}}$ and defined according to:

$D_{11}^{f}=\frac{1}{2} h d^{f}+D_{11}$

According to the number of end boundary conditions (14), with respect to the co-ordinate parameter $x$, the equations (3.15) would be a sixth order set of ordinary differential equations. These can be solved when a particular set of boundary conditions is specified at each end of the beam. It can be observed that, the difference between equations (3.15) and their counterparts, met in the case perfectly flexible fibers seen in the classical beam theory, is the definition of the rigidity $D_{11}^{f}$ shown in equation (18).

\section{Static Solution}

In the present section, the considered beam is subjected to under normal static load applied on its top surface. Therefore, the 1D static solution is found for the considered flexure problem. It can be resulted that the inertia terms appearing in the right-hand sides of the motion equations (17) are disregarded to yield the following equilibrium equations: 
$A_{11}^{c} u_{, x x}-B_{11}^{c} w_{, x x x}=0$,

$B_{11}^{c} u_{, x x x}-\left(D_{11}^{f}\right) w_{, x x x x}=q(x)$,

The lateral load function can be expressed in the following Fourier series.

$q(x)=q_{m} \sin \left(p_{m} x\right), p_{m}=m \pi / L_{1}, \quad(m=1,2, \ldots)$.

In addition, the following boundary conditions can be applied on the ends $x=0, L_{1}$ :

at a simply supported end: $N_{x}^{c}=0, w=0, M_{x}^{c}+M_{x}^{f}=0$,

at a clamped end: $u=0, w=0, w_{, x}=0$,

and at a free end: $N_{x}^{c}=0, M_{x, x}^{c}+M_{x, x}^{f}=0, M_{x}^{c}+M_{x}^{f}=0$,

The general solution of the non-homogeneous ordinary differential system (19) will be the tow degrees of freedom $u$, and $w$ and has the following form:

$S=S_{c}+S_{p}$.

where $S_{c}$ is the complementary solution and $S_{p}$ is a particular solution of the non-homogeneous system. The effective way to test the reliability of the presented theory is by performing numerical comparison with the corresponding results of the static plane strain asymmetric-stress elasticity solution found in[7] and, further, has been discussed in[3].In this subsection, the beam is taken as simply supported at the ends $x=0, L_{1}$.

The simply supported boundary conditions (21-a) are satisfied by the following trigonometric displacement choice of $S_{p}$ :

$u=A \cos \left(p_{m} x\right), w=C \sin \left(p_{m} x\right)$.

Inserting equations (23) into Navier-type differential equations (21) will convert it into the following system of tow simultaneous linear algebraic equations of the tow unknown constants $\mathrm{A}$ and $\mathrm{B}$.

$\left[\begin{array}{cc}p_{m}^{2} A_{11}^{c} & -p_{m}^{3} B_{11}^{c} \\ p_{m}^{3} B_{11}^{c} & -p_{m}^{4} D_{11}^{f}\end{array}\right]\left[\begin{array}{l}A \\ B\end{array}\right]=\left[\begin{array}{c}0 \\ q_{m}\end{array}\right]$

In order to find the values of A and B, the algebraic equations system (24) will be solved.For a homogeneous rectangular $(N=1)$ plate, Eq. (16-a) gives:

$B_{11}^{c}=0$.

Therefore, the Navier-type differential equations (19) reduce to the following uncoupled sets of differential equations:

$A_{11}^{c} u_{, x x}=0$,

$-\left(D_{11}^{f}\right) w_{, x x x x}=q(x)$,

The general solution of the sixth order equilibrium differential equations system (19) can be written as follows:

$u=\frac{k_{1}}{A_{11}^{c}} x+\frac{k_{2}}{A_{11}^{c}}+A \cos \left(p_{m} x\right)$,

$w=\frac{-k_{3}}{6 D_{11}^{f}} x^{3}-\frac{k_{4}}{2 D_{11}^{f}} x^{2}-\frac{k_{5}}{D_{11}^{f}} x-\frac{k_{6}}{D_{11}^{f}}+C \sin !\left(p_{m} x\right)$.

Values of the arbitrary constants $\left(K_{1}, K_{2}, \ldots, K_{6}\right)$ can be found when a set of six end boundary conditions is specified, see Eqs. (21). It should be noticed that as in perfectly flexible fibers case, thesearbitrary constants take zero value when SS boundary conditions are applied. This has as well been pointedoutin [8].It is worth mentioning the obtained general solution (27) is influenced the resistance of fibers in bending. This general solutioncanbe reduced to the perfectly flexible fibers solution by giving value of zero to the fiber bending elastic modulus $d^{f}$.Consequently, the value of the rigidity $D_{11}^{f}=D_{11}$ (see equation 18 ).

\section{Numerical Results and Discussion}

The following numerical results are for homogeneous transverse fiber-reinforced rectangular beams characterized by the following material properties[8]:

$E_{L} / E_{T}=40, \quad G_{L T} / E_{T}=0.5, \quad G_{T T} / E_{T}=0.2, v_{L T}=v_{T T}=0.25$.

Here, the subscript $L$ signifies the longitudinal fibers direction, $T$ denotes the transverse fiber direction, and $v_{L T}$ stands of the Poisson ratio that measure strain in the transverse direction $T$ under uniaxial normal stress in the $L$ direction. The elastic moduli shown in equations (5) and (7) are thus obtained by the use of equations (28) in conjunction with well-known related formulas (e.g. [6]). As pointed out in [4], the material property (28 a) characterizes the high stiffness ratio, such as, strong graphite-epoxy fibers, an appropriate value to the bending 
stiffness modulus of the fibers has to be assigned. In this regard and, in order to perform an appropriate comparison in the presented numerical results, the additional elastic $d^{f}$ is represented as follows:

$d^{f}=\frac{1}{12} C_{11} l L_{1}$,

whichis employed to include a material intrinsic length parameterl, which may, for instance, be considered related to the thickness or the diameter of the fiber. Furthermore, the following non-dimensional intrinsic material parameter:

$\lambda=\frac{l}{h}$

which is related the additional elastic $d^{f}$, and may be thought of as a fiber-to- plate or beam thickness. The normalized deflection employed for presentation of numerical results is defined according to:

$\bar{W}=E_{T} W / q_{1} L^{2}$

In the beginning of this section, the accuracy of the obtained solutions is assessed by comparing the results based on it to their counterparts based on the exact elasticity solution presented in [7] and [3]. After the reliability of the method has been checked, the effects of different sets of endboundary on the deflection of corresponding beams deformed by cylindrical bending. These boundary conditions are clamped-clamped end (CC), clamped-free end CF) and clamped-simply end (CS) boundary conditions.

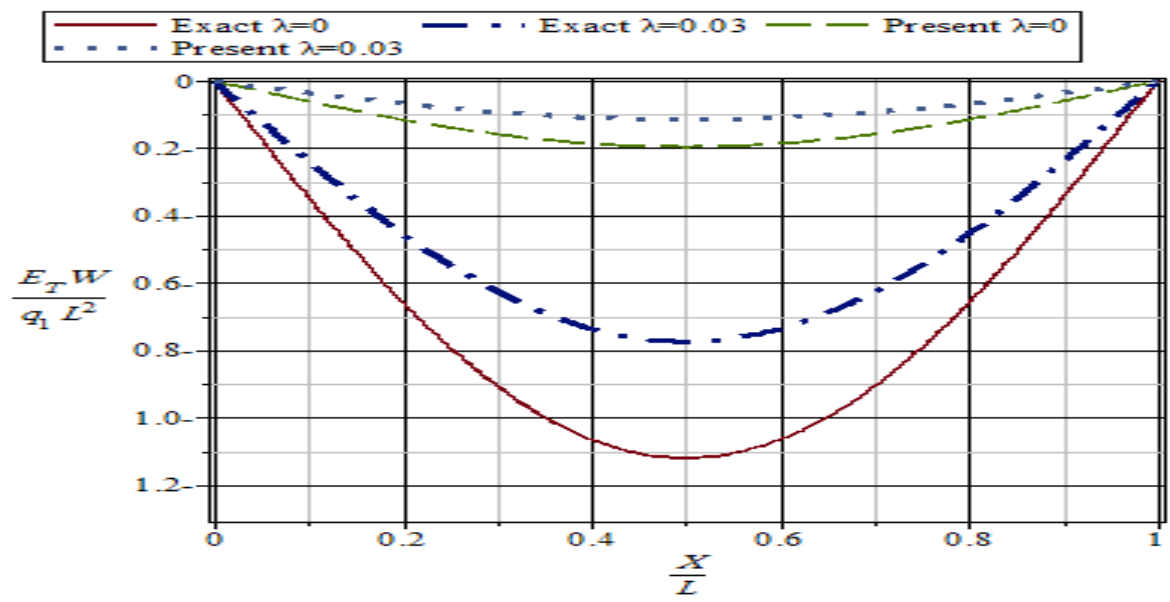

Figure 4. Deflection pattern along the $\mathrm{x}$-axis of a SS homogeneous beam

Figure 4 depicts and compares non-dimensional SS beam deflection and predicted by both the solution obtained in the present study and the corresponding exact polar elasticity solution [7] and [3] in which used as a benchmark in [4]. The beam is thin $(h / L=0.01)$ where the fiber runs along the $x$-direction. For comparison reason, the figure describes deflection not only for a case that fibers which have the bending stiffness $(\lambda=$ $0.03)$ but also for the case of the perfectly flexible $(\lambda=0)$.It is initially observed that, the differences between the deflection obtained based on the exact and the thin theory where $(\lambda=0)$ is very similar to that obtained based on the present solution presented in this study and the exact polar elasticity solution [7] and [3] where $(\lambda=0.03)$. It can be seen that, fiber bending resistance $(\lambda=0.03)$ reduces the beam deflection shown in the case of theperfectly flexible $(\lambda=0)$.

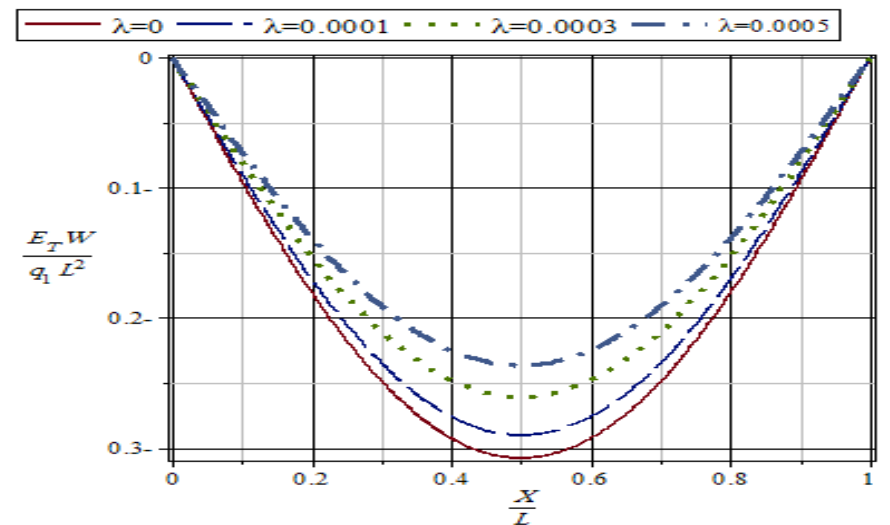

Figure 5.Deflection of a SS homogeneous beam at different values of $\lambda$ 
Figure 5 displays the through length distribution of $\bar{W}$ for the simply-simply supported (SS) beam at different values of $\lambda$. It is observed that by increasing the values of $\lambda$, the deflection of the beam decreases. This, as pointed out in [4], makes evident that the increase of the $\lambda$ valuesis equivalent to increasing the fiber bending stiffness. The curve which presents the largest values of deflection $(\lambda=0)$ is due to the case of perfectly flexible fibers.

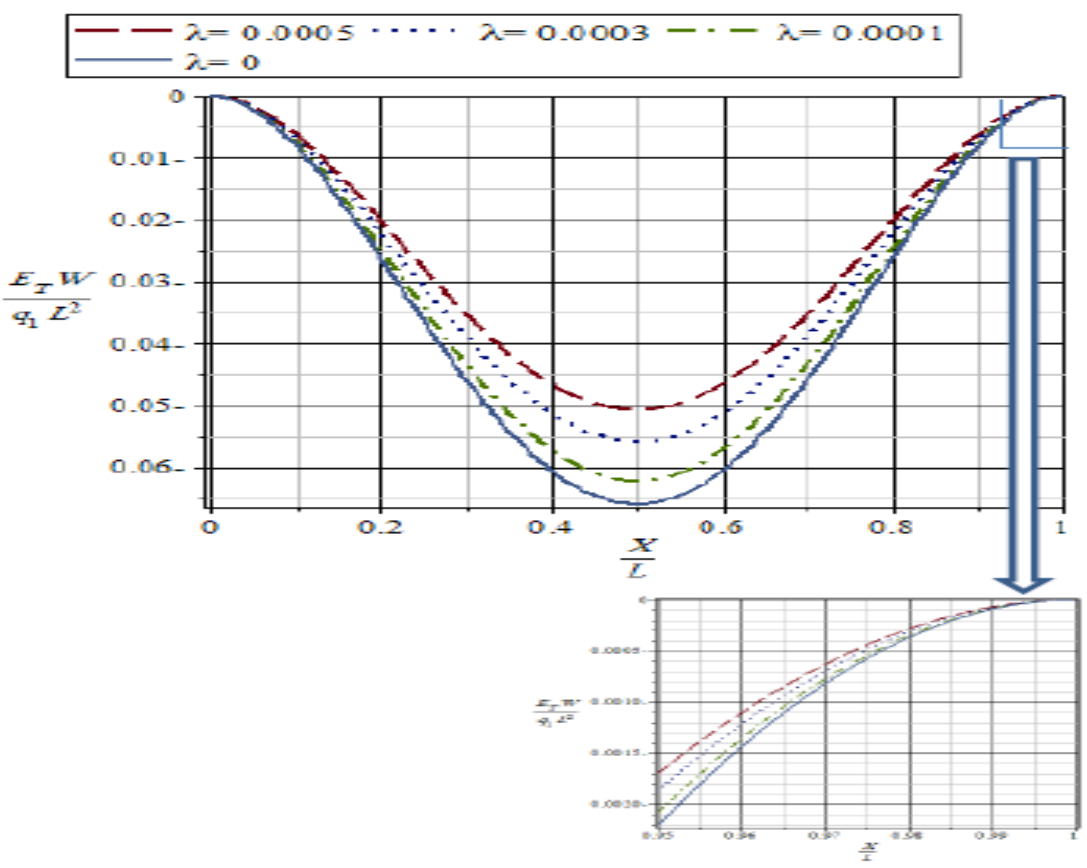

Figure6.Deflection of a CC homogeneous beam at different values of $\lambda$

Figure 6 shows the defection of a CC homogeneous beam at different values of $\lambda$. It is, again, seen that increasing the value of $\lambda$ reduces the beam deflection. Attention is drawn on the shape of the deflection at the fiber ends by presenting aseparate 'window' that lies underneath of the principal figure. It is observed that the effect of the clamped-clamped boundary conditions (21-b)becomes more evident where the beam ends are restrained against rotation. Furthermore,regardlessof whether the fibers are assumed perfectly flexible $(\lambda=0)$ or have the ability to resist bending $(\lambda=0.0001, \lambda=0.0003$ and $\lambda=0.0005)$, the maximum beam deflection of the clamped-clamped case is considerably smaller than that in the case of simply-simply supported beam (figure 5).

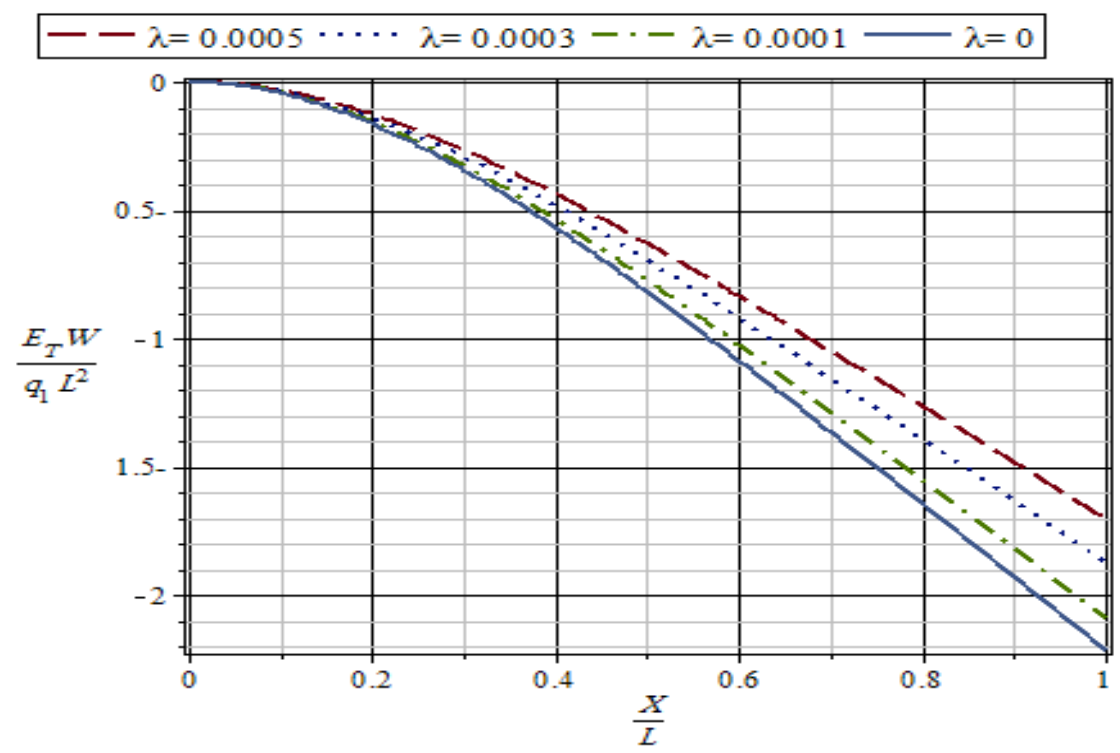

Figure 7. Deflection of a CF homogeneous beam at different values of $\lambda$ 
Figure 7 presents the deflection of a $\mathrm{CF}$ homogeneous beam at different values of $\lambda$ including the case of the perfectly flexible fibers $(\lambda=0)$. The deflection of this cantilevered beam is clearly seen decreasing when the value of $\lambda$ increses. This emphasise, again, that the increase of the $\lambda$ values is equivalent to increasing the fiber bending stiffness or, consequently, increasing the resistance of the beam bending.

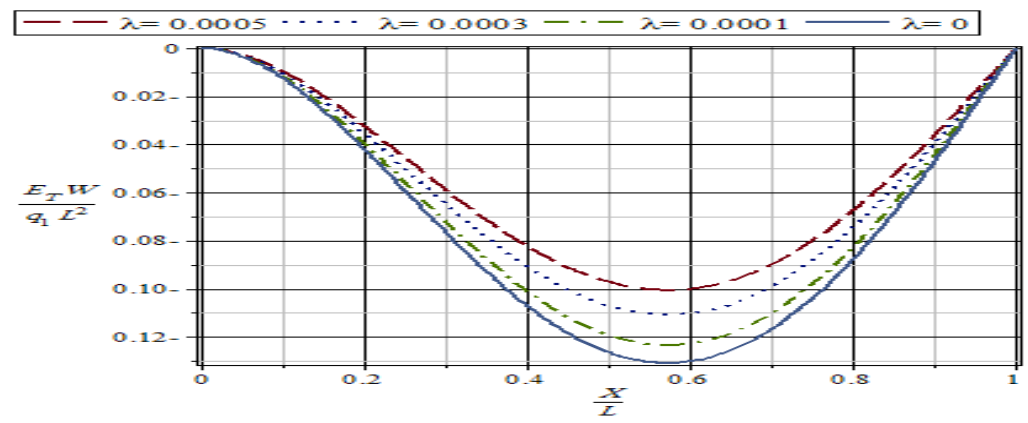

Figure 8. Deflection of a CS homogeneous beam at different values of $\lambda$

The deflection distribution through the length of theCS homogeneous beam is shown in figure 8 for the case of perfectly flexible fiber $(\lambda=0)$ and different cases of the presence of fiber bending stiffness $(\lambda=0.0001, \lambda=$ 0.0003 and $\lambda=0.0005)$. The difference of the effects of the boundary conditions can be seen clearly at the beamends. This is observed where the clamped beam end $(\mathrm{X} / L=0)$ is restrained against rotation while is not at the simply supported end $(\mathrm{X} / L=1)$. In addition, the maximum beam deflection of the clamped-simply case is smaller thanthat in the case of simply-simply supported beam, and beiger than that beam is clamped-clamped ends. It should be observed that, the presence of fiber bending stiffness $\lambda=0.0005$ results an approximately $23 \%$ reduction of the maximum non-dimensional deflection at the middle beam length $(\mathrm{X} / L=0.5$ ) (see fig.5, 6and 8).

\section{Conclusion}

Fiber bending stiffness is considered in the present study of Euler-Bernoulli beam theory. The displacement field of the classical beam theory is employed in the equations of motions which takes into account the ability of fibers to resist bending that have been presented in [2]. The static solutions of these equations of motions has been found in the present study where associated with an appropriate different sets of boundary equations that consider the fiber bending stiffness. Considerable numerical results have been presented for the static homogeneous beam bending problem where the beam is reinforced by fibers that resist bending. An attention is focused on the influence of the resistance of fibers in bending on the beam deflection. It has been evidently, as expected, shown that the increase of the fiber bending stiffness decreases the deflection of the beam. An attention has been drawn to the effect of the boundary conditions on the shape of deflection practically for the clamped beam ends where compared that with the simply-supported ends. Further study could be conducted on the other versions of plate theory considering the fiber bending stiffness such as Kirchhoff plate theory, Timoshenko beam theory and Higher-order theory.

\section{References}

[1] Spencer, A.J.M. and K.P. Soldatos, Finite deformations of fibre-reinforced elastic solids with fibre bending stiffness. International Journal of Non-Linear Mechanics, 2007. 42(2): p. 355-368.

[2] Soldatos, K.P., Towards a new generation of 2D mathematical models in the mechanics of thin-walled fibre-reinforced structural components. International Journal of Engineering Science, 2009. 47(11-12): p. 1346-1356.

[3] Farhat, A.F. and K.P. Soldatos, Cylindrical Bending and Vibration of Polar Material Laminates. Mechanics of Advanced Materials and Structures. 22(11): p. 885-896.

[4] K.P. Soldatos and A.F. Farhat, On Reissner's displacement field in modelling thin elastic plates with embedded fibres resistant in bending. IMA Journal of Applied Mathematics, 2016.

[5] Han, S.M., H. Benaroya, and T. Wei, Dynamics of transversely vibrayion beams using four engineering theories Journal of sound and vibration 1999. 225(5).

[6] Jones, R.M., Mechanics of composite materials. Hemisphere, New York., 1975

[7] Farhat, A.F., Basic problems of fibre reinforced structural components when fibres resist bending. PhD thesis, University of Nottingham 2013 Web. 24 February. 2017.

[8] Soldatos, K.P. and P. Watson, A method for improving the stress analysis performance of one- and two-dimensional theories for laminated composites. ActaMechanica, 1997. 123(1): p. 163-186.

Ali Farhat. "Euler-Bernoulli Beam Theory in the Presence of Fiber Bending Stiffness." IOSR Journal of Mathematics (IOSR-JM) 13.3 (2017): 10-17. 